in vivo $35: 3211-3220(2021)$

doi:10.21873/invivo.12616

\title{
Long Noncoding RNA ZFAS1 Promotes Progression of Oral Squamous Cell Carcinoma Through Targeting miR-6499-3p/CCL5 Axis
}

\author{
XIAOYONG QIU ${ }^{*}, \mathrm{CHENXI} \mathrm{LI}^{2,3,4,5^{*}}$ and HAO $\mathrm{CHEN}^{1}$ \\ ${ }^{1}$ Department of Stomatology, the Wuhan Sixth Hospital, Wuhan, P.R. China; \\ ${ }^{2}$ Department of Oral and Maxillofacial Surgery, Laboratory for Tumor Genetics and Regenerative Medicine, \\ University Hospital Hamburg-Eppendorf, Hamburg, Germany; \\ ${ }^{3}$ Department of Oral and Maxillofacial Oncology Surgery, \\ The First Affiliated Hospital of Xinjiang Medical University, Urumqi, P.R. China; \\ ${ }^{4}$ School of Stomatology, Xinjiang Medical University, Urumqi, P.R. China; \\ ${ }^{5}$ Stomatological Research Institute of Xinjiang Uygur Autonomous Region, Urumqi, P.R. China
}

\begin{abstract}
Background: Oral squamous cell carcinoma (OSCC) ranks sixth among malignancies in the world, and there are 200,000 new cases annually, rendering OSCC a significant global public health issue that has caused great burdens on patients, society, and the economy. Despite great progress in diagnosis and treatment methods, patient survival has not been greatly enhanced. Hence, there is an urgent need to identify novel targets that can serve as early diagnostic and therapeutic biomarkers for OSCC. Long non-coding RNAs (lncRNAs) participate in several cancer types, including OSCC. This work identified the competing endogenous RNA network related to IncRNA zinc finger nuclear transcription factor, X-box binding 1-type containing 1 antisense RNA 1 (ZFAS1) in OSCC, as well as the corresponding downstream targets. Materials and Methods: Firstly, we identified the IncRNA ZFAS1 levels in OSCC cells and tissues and confirmed its relationship to tumor progression. Secondly, we identified a lncRNA-miRNA-mRNA network, which was closely associated with OSCC development using bioinformatics methods. Next, our hypothesis that IncRNA ZFAS1 modulates OSCC progression was verified with in vitro and in vivo
\end{abstract}

This article is freely accessible online.

*These Authors contributed equally to this work.

Correspondence to: Hao Chen (ORCID: 0000-0003-1495-0739), Department of Stomatology, the Wuhan Sixth Hospital, No. 168 Hong Kong Road, Jiang'an District, Wuhan City 430015, Hubei Province, P.R. China. E-mail: 14995354@qq.com

Key Words: Oral squamous cell carcinoma, lncRNA, ZFAS1, ceRNA, CCL5. experiments. Results: Firstly, we found lncRNA ZFAS1 expression increased within OSCC cells and tissues and was positively associated with tumor progression. Secondly, its lncRNA-miRNA-mRNA network was determined, and the target of ZFAS1 was identified as miR-6499-3p/C-C motif chemokine ligand 5 (CCL5). Mechanistically, we found that ZFAS1 up-regulated CCL5 by competitively sponging miR6499-3p. Further studies demonstrated that ZFAS1 promoted tumor progression in vivo and in vitro. Conclusion: Our results indicate that ZFAS1 serves as a crucial oncogenic factor in OSCC occurrence and development and may therefore serve as a possible therapeutic target for OSCC.

Oral squamous cell carcinoma (OSCC) is a frequently seen type of head and neck cancer, which has an associated 5-year overall survival (OS) rate of about $50 \%$ (1). Surgical treatment, adjuvant radiotherapy, and chemotherapy remain the mainstay treatment methods of OSCC, but its 5-year OS rate (range $=45-50 \%$ ) has not markedly improved in the past 10 years $(2,3)$. Therefore, it is vital to find novel and effective therapeutic targets for OSCC so as to improve prognosis.

Long non-coding ribonucleic acids (lncRNAs) are RNAs over 200 nucleotides, which are not encoded to proteins. Recently, lncRNAs have been linked with OSCC genesis and development $(4,5)$. Competing endogenous RNAs (ceRNA), in which lncRNA competes for post-transcriptional control by sponging specific relevant miRNAs, is one of the known mechanisms through which lncRNAs act. Research has confirmed that microRNAs (miRNAs) also exert a vital role in regulating gene expression and cancer development (6). Therefore, identification of $\operatorname{lncRNA}-$ miRNA-mRNA regulatory axes might provide more clues to the molecular mechanisms involved in OSCC and potential therapeutic targets. 
LncRNA zinc finger nuclear transcription factor, X-box binding 1-type containing 1 antisense RNA 1 (ZFAS1), has been suggested to increase cancer promotion $(7,8)$. However, to our knowledge, there has been no investigation of the role of ZFAS1 in OSCC yet. C-C Chemokine ligand 5 (CCL5) has been identified to exert a pivotal part in immune surveillance, inflammation, and cancer development $(9,10)$. As a result, this work focused on exploring the role of lncRNA ZFAS1 in OSCC and elucidating the mechanisms involved.

\section{Materials and Methods}

Patients and specimens. Twenty patients with a confirmed histological diagnosis of OSCC were enrolled from the Wuhan Sixth Hospital. None of the cases received preoperative neoadjuvant therapy. Their formalin-fixed, paraffin-embedded OSCC samples and matched non-carcinoma tissue samples were utilized for detecting the lncRNA ZFAS1 level. The Ethics Committees of the Wuhan Sixth Hospital approved the project protocol (IRB-20-03801). Each case provided informed consent for participation.

Cell lines and culture. The OSCC cell lines TSCCA, Tca8113, SCC-9, CAL-27, and normal human oral keratinocytes (NHOK) were purchased from the American Type Culture Collection (Manassas, VA, USA), cultured in RPMI 1640 medium or Dulbecco's modified Eagle's medium (HyClone, Thermo Fisher Scientific Inc., Waltham, MA, USA) that contained $10 \%$ fetal bovine serum (FBS; HyClone), followed by incubation in a humidified incubator at $37^{\circ} \mathrm{C}$ with $5 \% \mathrm{CO}_{2}$.

Vector construction and cell transfection. Small interfering RNA (siRNA) against LncRNA ZFAS1 (si-ZFAS1: 5'-GCTGCTCGAGA CTACATTT-3') and matched control si-NC (5'-UUCUCCGAAC GUGUCACGUTT-3') were constructed by RiboBio (Guangzhou, PR China). Transfection was carried out by adopting OPTI-MEM and Lipofectamine 3000 (Invitrogen) in accordance with specific protocols. Then cells in the logarithmic growth phase $\left(3 \times 10^{4} /\right.$ well $)$ were harvested and plated into 6 -well plates. At $48 \mathrm{~h}$ posttransfection, RNA reverse transcription and quantitative real-time polymerase chain reaction (qRT-PCR) were performed to assess silencing efficiency. $m i R-6499-3 p$ mimics, miRNA negative control, lentivirus overexpressing $C C L 5$, and control vector were also purchased from RiboBio, and the same transfection procedure was performed.

$q R T-P C R$. Trizol kit (Thermo Fisher Scientific Inc., Waltham, MA, USA) was adopted for extracting total RNA from tissues and cells. Ultraviolet spectroscopy was used to measure RNA purity and concentration. Thereafter, cDNA was prepared from the extracted total RNA using miRNA qPCR Quantitation Kit (GenePharma, Shanghai, PR China) and PrimeScript RT Reagent Kit (Takara, Japan) through reverse transcription following specific protocols. The StepOnePlus ${ }^{\mathrm{TM}}$ System (Applied Biosystems, Foster City, CA, USA) was utilized for qRT-PCR by adopting the SYBR Green Master Mix (Takara, Japan), with U6 and glyceraldehyde 3phosphate dehydrogenase $(G A P D H)$ as the internal references for miRNA and mRNA determinations, respectively. The relative quantification method $\left(2^{-\Delta \Delta C t}\right)$ was used to calculate fold changes of cDNA. PCR assay was performed for each sample with three duplicates and repeated three times.
Western blot analysis. After transfection for $48 \mathrm{~h}$, and total protein was extracted and measured by the bicinchoninic acid approach. After sodium dodecyl-sulfate polyacrylamide gel electrophoresis using 50 $\mu \mathrm{g}$ of protein, proteins were transferred to a polyvinylidene fluoride membrane (Millipore, Billerica, MA, USA). Membranes were blocked with 5\% skimmed milk for $1 \mathrm{~h}$ under ambient temperature, followed by incubation using primary monoclonal antibodies to: CCL5 (AF5151; Affinity Biosciences, Cincinnati, OH, USA) and GAPDH (5174; Cell Signaling Technology, Boston, MA, USA) overnight at $4^{\circ} \mathrm{C}$. The membrane was incubated with secondary antibody was horseradish peroxidase-linked anti-rabbit IgG (7074, Cell Signaling Technology). After that, the ECL Plus Western Blotting Detection System (Amersham Biosciences) was employed for visualization. Finally, Image-Pro Plus 6.0 software (Media Cybernetics, USA) was utilized for quantifying band intensities.

CCK8. After incubation in 6-well plates for a period of $24 \mathrm{~h}$, transfected TSCCA and CAL-27 cells were subjected to trypsin digestion before they were seeded into 96 -well plates (4,000/well). Cell growth was analyzed at $0,24,48$ and $72 \mathrm{~h}$. Cell viability was measured using CCK-8 (Dojindo, Tokyo, Japan) in line with the manufacturer's instructions. In brief, CCK8 solution $(10 \mu \mathrm{l})$ was added to every well, followed by incubation of cells for $2.5 \mathrm{~h}$. Thereafter, a microplate reader (Tecan Trading AG; Männedorf, Switzerland) was employed for measuring OD values at $450 \mathrm{~nm}$.

Colony-formation assay. Transfected cells in the logarithmic phase were resuspended in medium and plated into culture plates. They were cultured for an additional $2-3$ weeks at $37^{\circ} \mathrm{C}$ with $5 \% \mathrm{CO}_{2}$ until cell colonies were observed with naked eye. Cells were then rinsed and fixed using $4 \%$ paraformaldehyde for $15 \mathrm{~min}$. Cells were then stained with Giemsa (Dinguo, PR China) for approximately 15 min. After drying, the colonies formed were counted under a microscope, and the colony-formation rate was determined.

Scratch assay. For scratch assays, transfected cells were plated in 6 -well plates and incubated until reaching $100 \%$ cell confluency. Cell culture-insert (ibidi, 80206) was adopted for scratch assay. Later, the original medium was substituted with medium containing $10 \%$ FBS. An Olympus microscope was utilized to record the scratch size at $0 \mathrm{~h}$. At $24 \mathrm{~h}$ later, the scratch size was measured at the same site. The closure rate was then determined by the following formula (scratch size at $0 \mathrm{~h}-$ scratch size at $24 \mathrm{~h}$ )/scratch size at $0 \mathrm{~h} \times 100 \%$.

Transwell assay. Transwell chambers (pore diameter, $8 \mu \mathrm{m}$; Costar; Corning Incorporated, Corning, NY, USA) were used for Transwell assay. Matrigel (356234, BD Biosciences, Eysins, Vaud, Switzerland) was coated onto Transwell chambers beforehand in accordance with the manufacturer's instructions. At $48 \mathrm{~h}$ posttransfection, cells were collected and resuspended in the serum-free medium into a single-cell solution. Afterwards, the upper Transwell chamber was added $1 \times 10^{5}$ cells in a serum-free medium $(200 \mu \mathrm{l})$, and $500 \mu \mathrm{l}$ medium containing $20 \%$ FBS as the chemoattractant was added into the lower chamber. After incubation for $24 \mathrm{~h}, 100 \%$ methanol was applied for $5 \mathrm{~min}$ for cell fixation, then $0.5 \%$ crystal violet (Beyotime Institute of Biotechnology, Haimen, PR China) was used to stain cells for an additional $5 \mathrm{~min}$. A cotton swab was then used to remove the non-invading cells gently. Then, under microscopy (CKX41; Olympus Corporation, Tokyo, Japan), five 
A

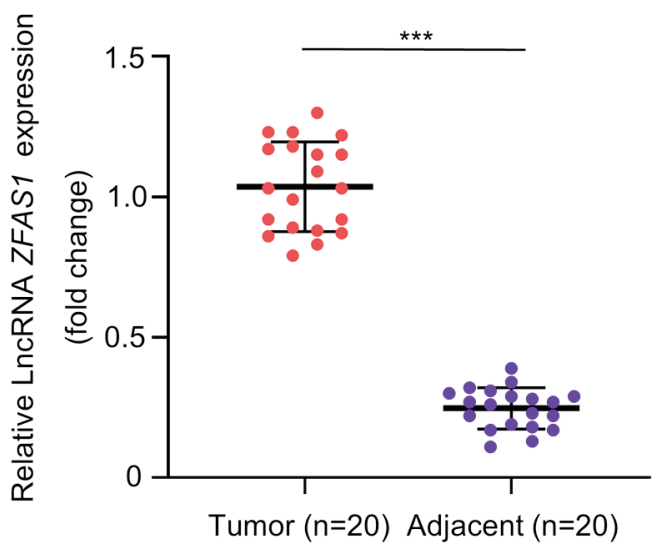

B

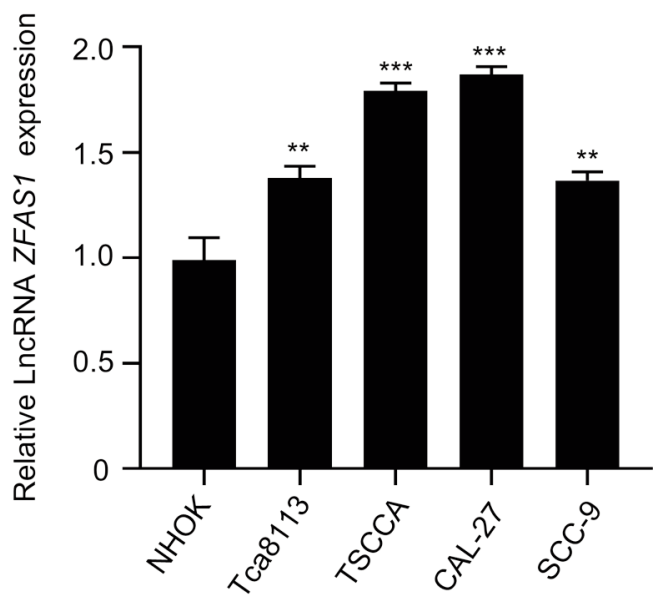

Figure 1. Long non-coding RNA (lncRNA) zinc finger nuclear transcription factor, X-box binding 1-type containing 1 antisense RNA 1 (ZFAS1) is up-regulated in oral squamous cell carcinoma (OSCC) tissues and cell lines. A: mRNA expression of lncRNA ZFAS1 was elevated in tumor tissues relative to adjacent non-carcinoma tissues from patients with OSCC. B: mRNA expression of ZFAS1 was elevated in OSCC cell lines compared to normal human oral keratinocytes $(N H O K)$. Significantly different at: **p<0.01 and ***p<0.001.

random fields were selected from every membrane for counting the invading cells.

Bioinformatics analysis. The putative miRNA-binding sites of ZFAS1 were predicted with bioinformatic analysis using the online bioinformatics website DIANA TOOLS (http://diana.imis.athenainnovation.gr/DianaTools/index.php). The potential interaction sites between miRNAs and mRNAs were obtained using TargetScan (http://www.targetscan.org)

Dual-luciferase reporter gene assays. HEK293T cells were cultivated in a 12-well plate until reaching $80-90 \%$ confluency. The luciferase reporter plasmids, which included mutant-type (Mut) and wild-type (WT) pGL3-ZFAS1-3'UTR, together with Mut and WT pGL3-CCL5-3'UTR, were prepared by GenePharma (Shanghai, PR China). After that, PGL3-CCL5-3'-UTR Mut or WT, and PGL3CCL5-3'-UTR Mut or Wt were co-transfected with miR-6499-3p mimics or NC mimics into HEK293T cells by the use of Lipofectamine ${ }^{\circledR} 3000$ (Thermo Fisher Scientific Inc.), following the manufacturer's instructions. At $48 \mathrm{~h}$ post-transfection, the DualLuciferase Reporter Assay System (Promega Corporation) was applied to measure the luciferase activity. In every transfected well, the firefly luciferase activity was normalized to that of Renilla. Every experiment was repeated thrice.

Anti-Argonaute-2 (AGO2) RNA-binding protein immunoprecipitation assay (RIP) assay. miR-6499-3p or miR-NC was used to transfect OSCC cells. At $48 \mathrm{~h}$ post-transfection, transfected cells were subjected to RIP assays using Magna RIP ${ }^{\text {TM }}$ RNA Binding Protein Immunoprecipitation Kit (Millipore). Thereafter, magnetic beads bound to $\mathrm{NC} \operatorname{IgG}$ (Millipore) or anti-AGO2 (Millipore) were used to incubate cells, and qRT-PCR was conducted for measuring the relative level of ZFAS1 in the immunoprecipitated RNAs.

In vivo tumorigenicity assay. pSilencer4.1-CMV-puro-ZFAS1 plasmid was transfected into cells to obtain stable ZFAS1-deficient cells. After that, BALB/c mice (6 weeks old) were injected in the right-back with $5 \times 10^{6} \mathrm{CAL}-27$ cells with stable transfection of ZFAS1-silencing or control retroviral vectors in $250 \mu \mathrm{l}$ PBS. Altogether seven animals were enrolled in each group. We recorded tumor size at intervals of 3 days by measuring two perpendicular diameters of each implant using calipers. In addition, we determined tumor volume $\left(\mathrm{cm}^{3}\right)$ as follows, volume $\left(\mathrm{mm}^{3}\right)=1 / 2 \times$ length $\times$ width $^{2}$. At 6 weeks after injection of transfected cells, we sacrificed the mice bearing tumors. We then collected the tumors to evaluate the growth of the lesions, followed by determining the expression levels of lncRNA-miRNA-mRNA networks with qRT-PCR. Each animal experiment was conducted in line with the ethical guidelines from the Wuhan Sixth Hospital.

Statistical analysis. Statistical analyses were completed by SPSS17.0 (SPSS Inc., Chicago, IL), while statistical graphing was accomplished with GraphPad Prism 5 (GraphPad, San Diego, CA, USA). Data are displayed as the mean $\pm \mathrm{SD}$ and were compared between different groups by $t$-test. A difference of $p<0.05$ indicated statistical significance.

\section{Results}

LncRNA ZFAS1 is up-regulated in OSCC cell lines and tissues. This study determined the ZFAS1 level in 20 OSCC tissue samples and 20 matched non-carcinoma samples. As revealed by qRT-PCR, ZFAS1 expression was increased in OSCC tissues compared with matched normal tissue (Figure 1A). Moreover, ZFAS1 expression in OSCC cells (TSCCA, Tca8113, SCC-9, and CAL-27) was also significantly higher than in normal human oral keratinocyte cell line (NHOK) by qRT-PCR (Figure 1B). Thus, these results strongly suggest that ZFAS1 might participate in OSCC genesis and progression. 
ZFAS1 silencing inhibits OSCC cell proliferation migration and invasion in vitro. To further investigate the effect of ZFAS1 on OSCC, the loss-of-function method was used to silence ZFAS1 expression in TSCCA and CAL-27 OSCC cells. Forty-eight hours after siRNA transfection, the silencing efficiency of si-ZFAS1 was determined by qRTPCR, which revealed that ZFAS1 expression decreased by about $50 \%$ in si-ZFAS1-transfected cells relative to sh-NCtransfected cells (Figure 2A). Following the knockdown of LncRNA ZFAS1 in TSCCA and CAL-27 cells, CCK8 assays were performed for investigating the function of ZFAS1 in the proliferation of cancer cells. As a result, ZFAS1 silencing remarkably suppressed TSCCA and CAL27 cell proliferation (Figure $2 \mathrm{~B}$ ). Furthermore, the function of ZFAS1 in the proliferation of CAL-27 and TSCCA cells was evaluated by colony-formation assay, which came to consistent results (Figure 2C), with remarkable reduction of cancer cell colonies being seen in the experimental group relative to the control group $(p<0.001)$. This result confirmed si-ZFAS1 inhibited colony formation of OSCC cancer cells.

In addition, for evaluating the effect of ZFAS1 knockdown in TSCCA and CAL-27 cell migration, scratch assays were performed on both cell lines. Figure 2D shows that ZFAS1 silencing inhibited the migration of TSCCA and CAL-27 cells. Alterations of cell invasion were also determined by Transwell assays. Similar to the above results, knockdown of ZFAS1 inhibited invasion of OSCC cells (Figure 2E). All the experiment results substantiate the possibility that ZFASI promotes the progression of OSCC.

LncRNA ZFAS1 acts as an effective miRNA-6499-3p sponge in OSCC. Figure 3A shows the results of online bioinformatics website DIANA TOOLS in estimating the potential role of ZFAS1 as the sponging ceRNA for $m i R-6499-3 p$ and responsible for down-regulation of its expression. To confirm this interaction, luciferase reporter plasmids containing ZFASI coding sequences possessing binding sites for Mut or WT miR-6499-3p were built to identify the target effectors. Following plasmid transfection into TSCCA and CAL-27 cells, we found that miRNA-6499-3p mimics reduced luciferase activity of WT reporter vector, indicating the negative correlation between IncRNA ZFAS1 and miR-6499$3 p$ (Figure 3B).

Generally, lncRNA binds to the miRNA response elements in the RNA-induced silencing complex, and AGO2 protein is an essential part of this complex. Therefore, the anti-AGO2 RIP assay was conducted in this study, in which antibody to AGO2 was utilized to immunoprecipitate $m i R-6499-3 p$ and ZFAS1 in CAL-27 and TSCCA cell lysates. We found that the amount of accumulated ZFAS1 binding to AGO2 protein of $m i R-6499-3 p$ mimic group was significantly increased compared with the NC group (Figure 3C). Consistent with the above results, when performing qRT-PCR 48 hours after transfecting si-ZFAS1 and si-NC into CAL-27 and TSCCA cells, miRNA-6499-3p expression was significantly upregulated in si-ZFAS1-transfected cells compared with si-NCtransfected cells (Figure 3D). The above findings suggest the role of ZFAS1 as the sponging ceRNA for miR-6499-3p, which reduced the miR-6499-3p level in OSCC.

CCL5 serves as a direct target of miRNA-6499-3p. Using the online analysis tool (TargetScan, http://www.targetscan.org ), the target gene of $m i R-6499-3 p$ was predicted to be CCL5 (as shown in Figure 3E), which was reported to participate in the genesis and progression of different human cancer types (9-11).

Next, we constructed luciferase reporter plasmids that possessed CCL5 mRNA 3'-UTRs that contained mutations of miRNA-6499-3p-binding sites (Mut) and WT CCL5 mRNA. As a result, WT plasmid markedly reduced the luciferase activity in miRNA-6499-3p mimic-transfected cells relative to miR-NC-transfected cells (Figure $3 \mathrm{~F}$ ). In contrast, the luciferase activity of mutant plasmid did not differ between cells treated with miRNA-6499-3p mimics and miRNC (Figure 3F). These results confirmed that miRNA-6499$3 p$ inhibits $C C L 5$ expression through direct combination with the CCL5 mRNA 3'-UTR.

Moreover, qRT-PCR and western blotting assay verified that CCL5 expression decreased at both the RNA and protein levels in miRNA-6499-3p mimic-transfected CAL-27 and TSCCA cells compared with cells transfected with mimic control (Figure $3 \mathrm{G}$ and $3 \mathrm{H}$ ). In summary, this study found that $m i R-6499-3 p$ negatively regulated CCL5 expression.

miRNA-6499-3p/CCL5 mediate the effects of IncRNA ZFAS1 on TSCCA and CAL-27 cells. For investigating the relationship between the function of 1ncRNA ZFAS1 and the impacts of miRNA-6499-3p on CCL5 gene transcription, a rescue assay was carried out using down-regulation of miRNA-6499-3p or up-regulation of CCL5 in cells with reduced ZFAS1 expression. Plasmids and RNAs were transfected into TSCCA and CAL-27 cells, and then all assays were carried out 48 hours after transfection. CCK 8 assay result suggested that ZFAS1 silencing reduced cell proliferation, which was restored when cells were cotransfected with miRNA-6499-3p inhibitor or CCL5 vector (Figure 4A). In addition, the clone formation assay, the scratch experiment, and the transwell assay also showed that knockdown of ZFAS1 reduced the cloning-formation ability, migration, and invasion capacity of cells. However, these traits were rescued or partially recovered by co-transfection with miRNA-6499-3p inhibitor or CCL5 vector (Figure 4BD). To sum up, CCL5 expression and miR-6499-3p inhibition restored the tumor-promoting activity of lncRNA ZFAS1 in OSCC cells. 

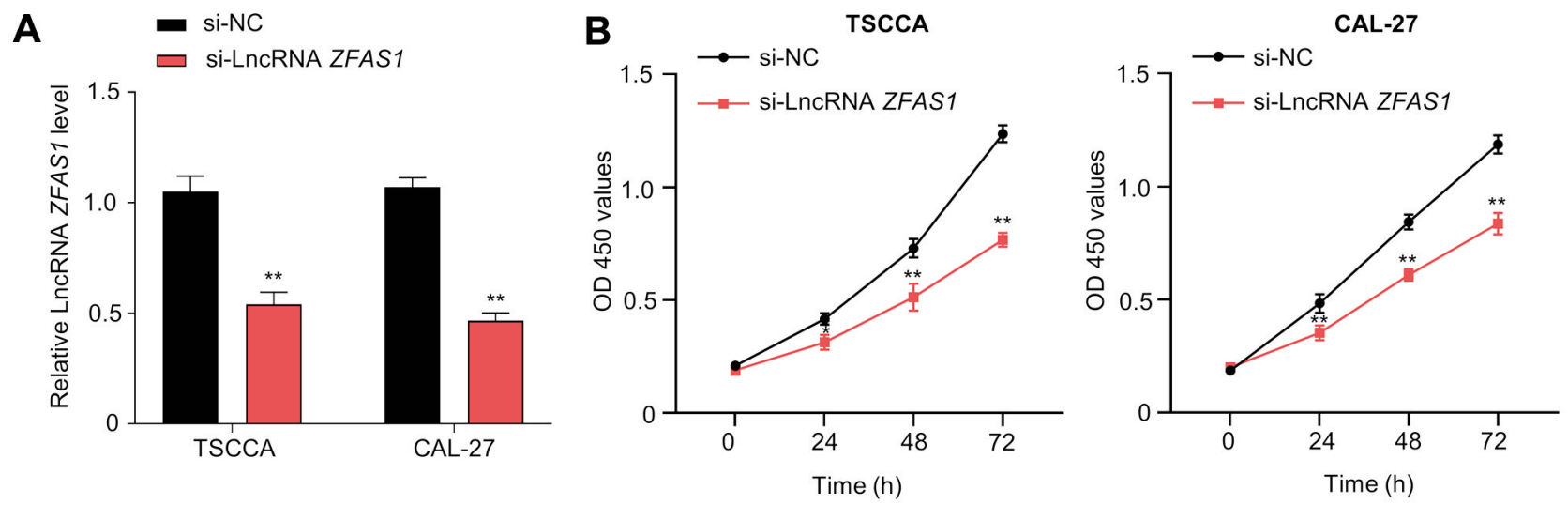

C
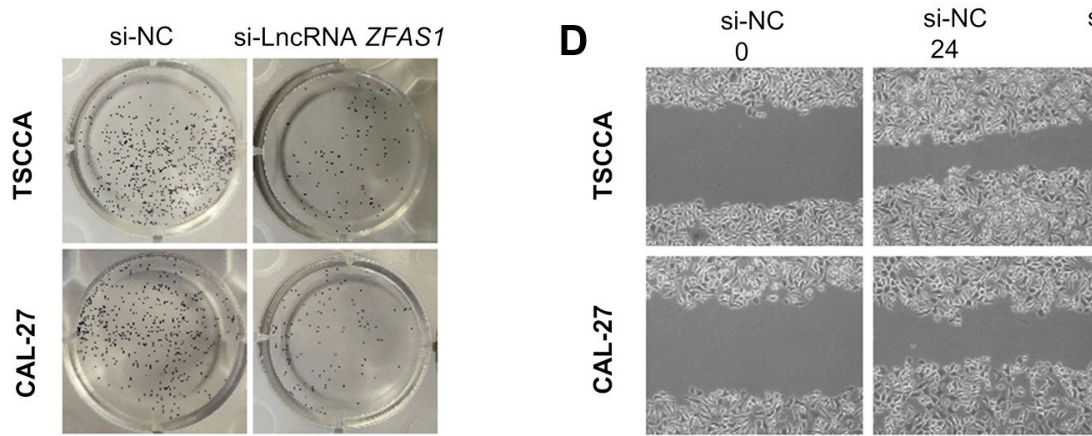

si-LnCRNA ZFAS1 si-LncRNA ZFAS1

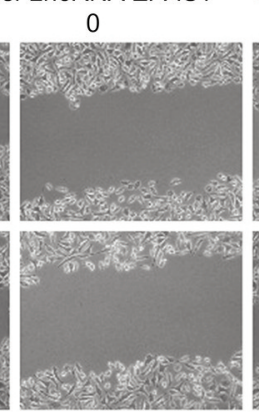

24
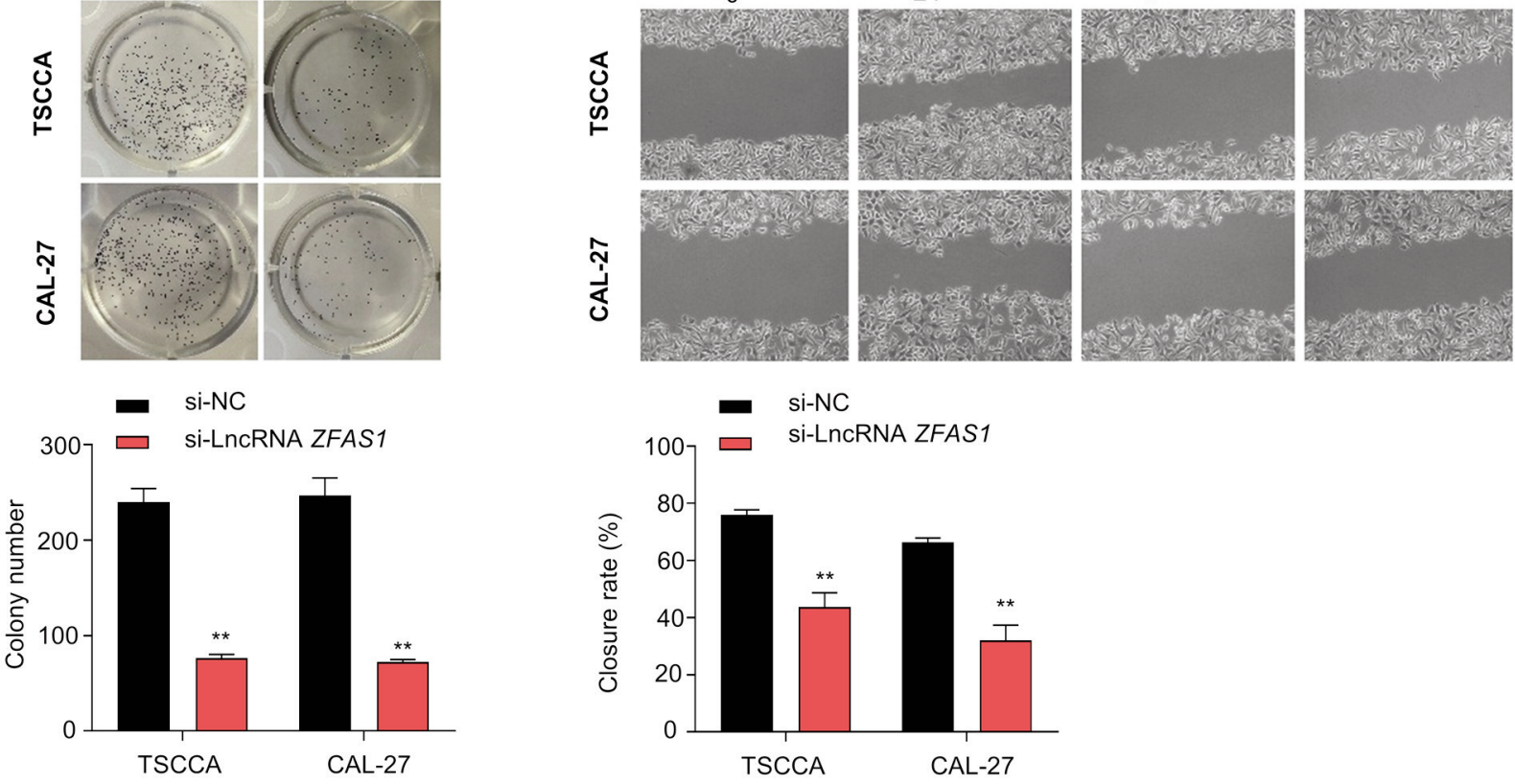

\section{E}
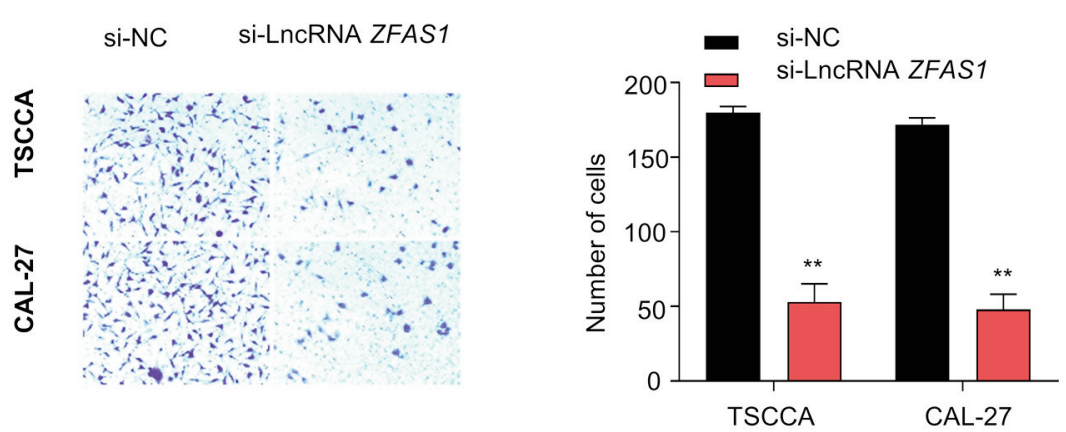

Figure 2. A: In oral squamous cell carcinoma (OSCC) cells, small-interfering Iong non-coding RNA (si-lncRNA) zinc finger nuclear transcription factor, X-box binding 1-type containing 1 antisense RNA 1 (ZFAS1) down-regulated ZFAS1 expression in comparison with si-negative control (NC). B: si-lncRNA ZFAS1 markedly inhibited the proliferation of TSCCA and CAL-27 cells. C: ZFAS1 inhibits clone formation of OSCC cancer cells. D: si-IncRNA ZFAS1 suppressed migration of OSCC cells. E: si-IncRNA ZFAS1 suppressed invasion of OSCC cells. **Significantly different at p<0.01. 
A

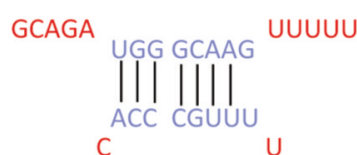

C

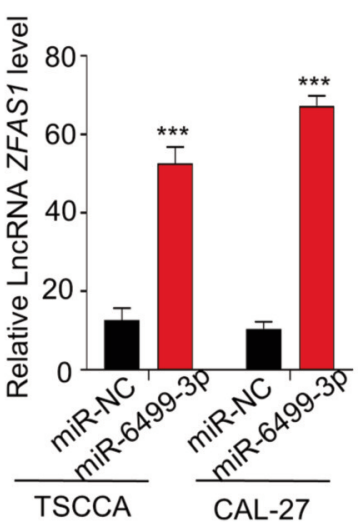

$\mathbf{F}$

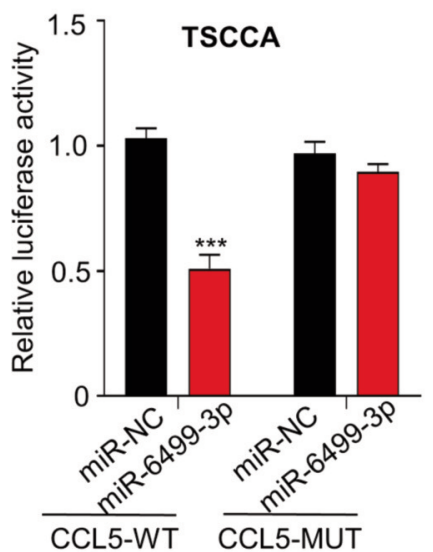

D
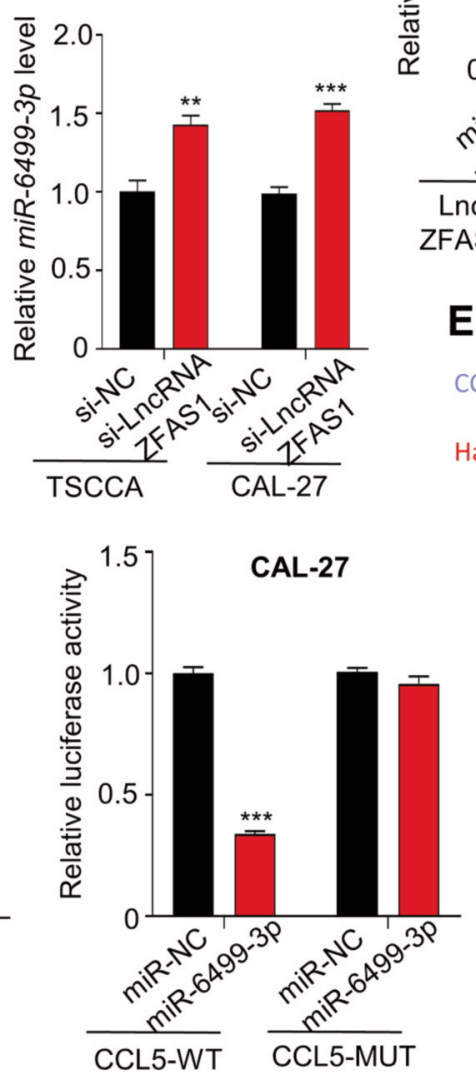

E
TSCCA

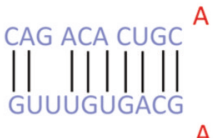

B
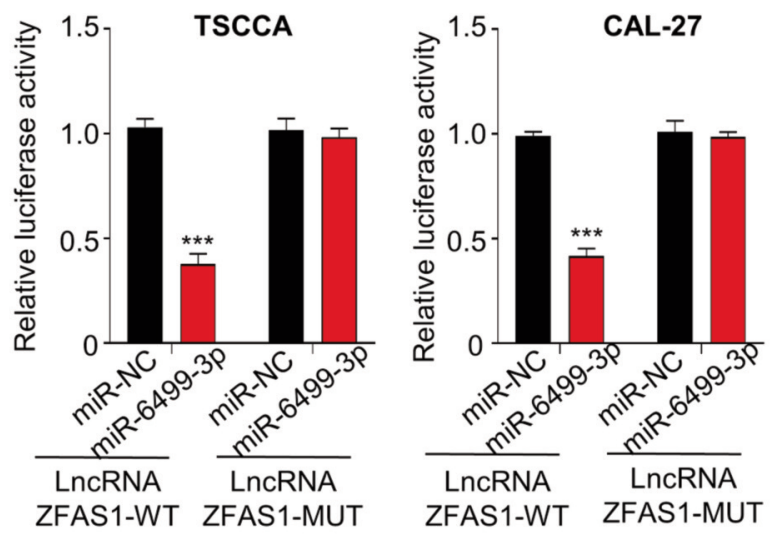

CCL5 3' UTR : 5' GAGCCGAGAUCGCGC CACUGCAC 3'

Has-miR-6499-3p: 3' ACACCCGUUUUGUUUGUGACGA 5'

H

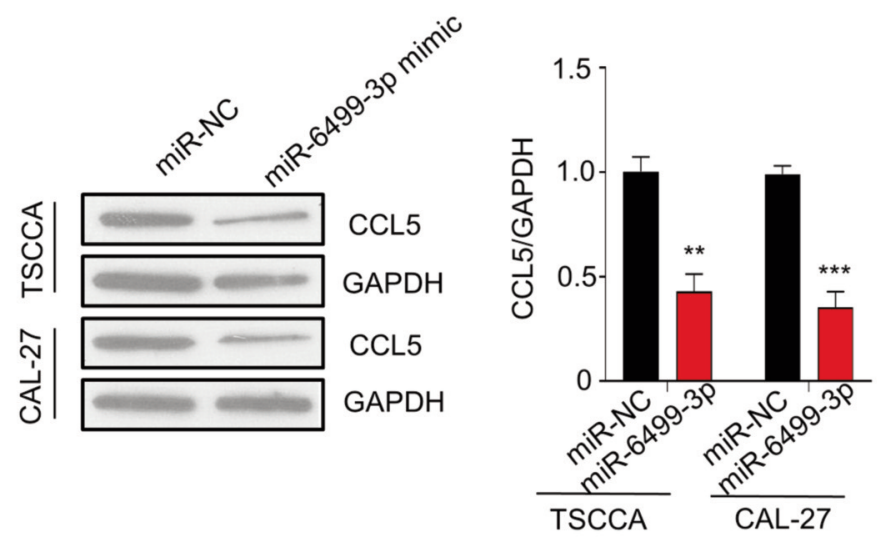

Figure 3. A: Predicted binding sites for long non-coding RNA (lncRNA) zinc finger nuclear transcription factor, X-box binding 1-type containing 1 antisense RNA 1 (ZFAS1), and miR-6499-3p using the DIANA bioinformatics tool (http://diana.imis.athena-innovation.gr/DianaTools/index.php). B: Relative luciferase activity in luciferase reporter plasmids, which included mutant-type (Mut) and wild-type (WT) pGL3-ZFAS1-3'UTR. Activity in pGL3-ZFAS1-Mut-transfected cells was not suppressed by miR-6499-3p. C: There was much more accumulated ZFAS1 binding to Argonaute 2 (AGO2) protein in the miR-6499-3p mimic group. D: Expression of miRNA-6499-3p was up-regulated in si-lncRNA ZFAS1 cells. E: The predicted binding site for C-C motif chemokine ligand 5 (CCL5) and miR-6499-3p by bioinformatics (http://www.targetscan.org). F: Relative luciferase activity in pGL3-CCL5-Mut-transfected cells was not suppressed by miRNA-6499-3p. G: and H: In cells transfected with miRNA-6499-3p mimic, expression of both CCL5 RNA and protein were reduced. Significantly different at: $* * p<0.01$ and $* * * p<0.001$. 
A

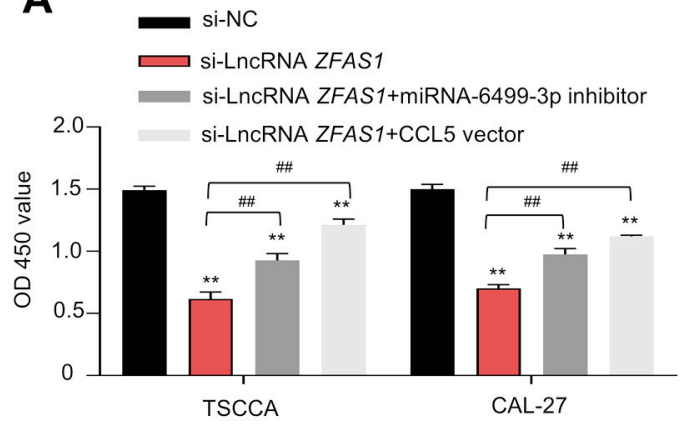

B

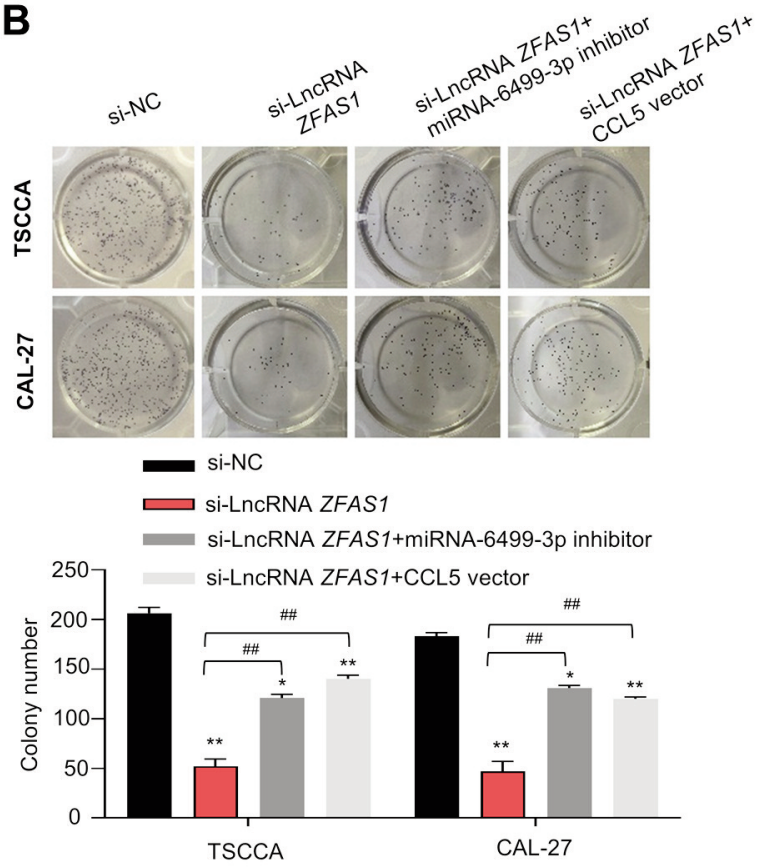

C
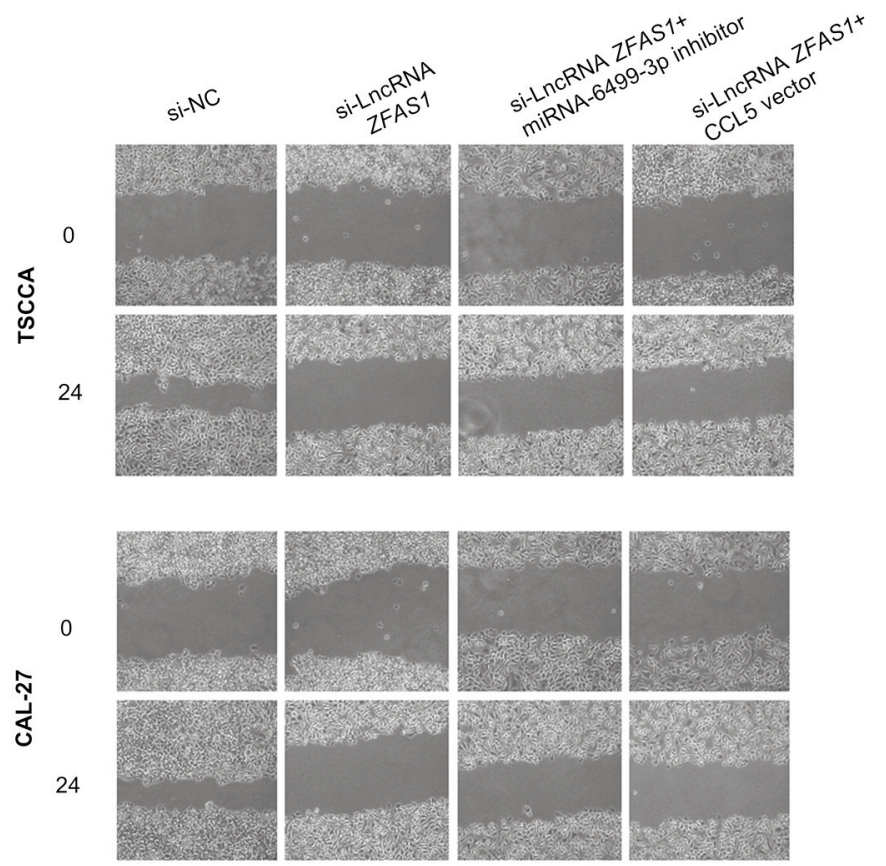

D

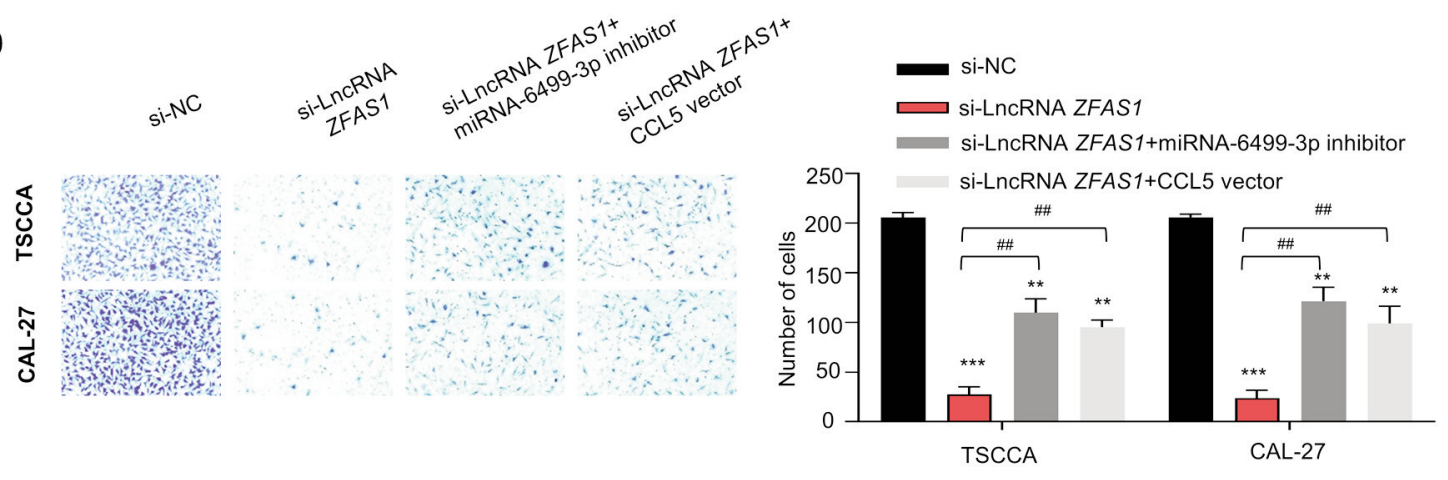

Figure 4. A: miRNA-6499-3p inhibitor and C-C motif chemokine ligand 5 (CCL5) vector reduced the inhibition of proliferation under silencing of long non-coding RNA (lncRNA) zinc finger nuclear transcription factor, X-box binding 1-type containing 1 antisense RNA 1 (ZFAS1) with small interfering RNA (si-RNA). Effects of miRNA-6499-3p inhibitor and CCL5 vector on the inhibitory effect of lncRNA ZFAS1 silencing in colony formation (B), migration $(C)$, and invasion $(D)$ by cells. Significantly different at: $* p<0.05$, **\#\# $p<0.01$ and $* * * p<0.001$. 
A

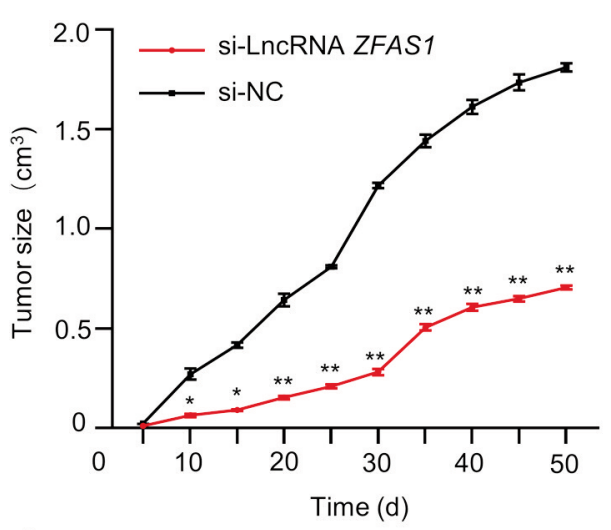

B

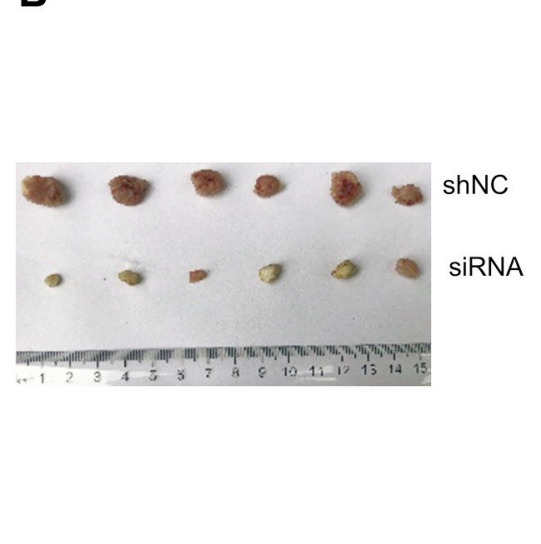

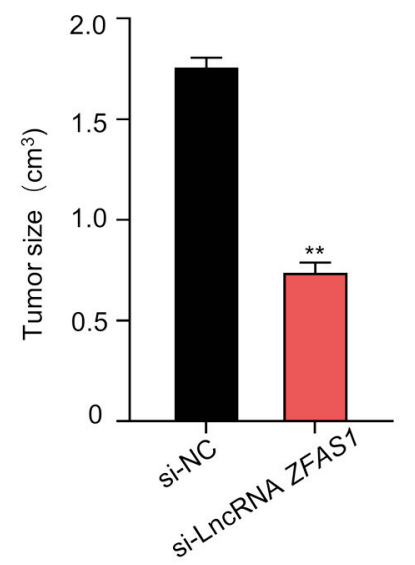
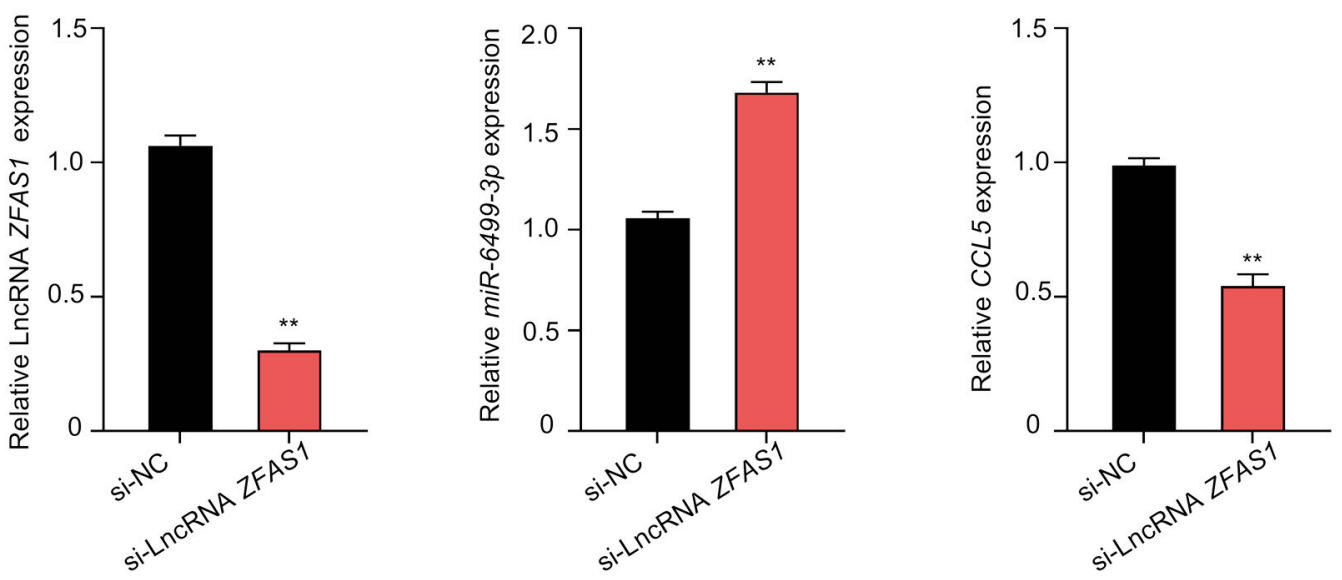

Figure 5. In vivo tumorigenicity assay in BALB/c mice injected in the right-back with CAL-27 cells with stable transfection of long non-coding RNA (lncRNA) zinc finger nuclear transcription factor, X-box binding 1-type containing 1 antisense RNA 1 (ZFAS1)-silencing (si) or control (NC) retroviral vectors. A: IncRNA ZFAS1-knockdown slowed tumor growth in vivo. B: LncRNA ZFAS1-knockdown reduced the terminal tumor volume. $C$ : miR-6499-3p and C-C motif chemokine ligand 5 (CCL5) levels in tumor tissues. Significantly different at: * $p<0.05$ and $* * p<0.01$.

Inhibition of ZFAS1 suppressed the proliferation of OSCC cells in vivo. For better determining ZFAS1 function in OSCC progression in vivo, CAL-27 cells with stably silenced ZFAS1 were established and injected into nude mice subcutaneously. We recorded tumor volume at intervals of 3 days after tumor formation and removed these tumors after 6 weeks. As a result, compared with control sh-NC mice, tumor volume was markedly lower in mice injected with CAL-27 cells with shRNA-mediated stable ZFAS1 knockdown (Figure 5A). Moreover, the final tumor volume was markedly lower after ZFAS1 was down-regulated in OSCC cells (Figure 5B). We conducted qRT-PCR for the expression of 1ncRNA ZFAS1, miR-6499-3p, and CCL5 in tumors. Consistent with the results of our previous experiments in si-ZFASl cells, miR-6499-3p expression was elevated, and CCL5 expression was down-regulated in tumor tissues (Figure 5C). Therefore, the above findings revealed that ZFAS1 suppressed tumor development in vivo by targeting the miRNA-6499-3p/CCL5 axis.

\section{Discussion}

LncRNAs have been recently proposed to participate in the pathogenic mechanisms of many kinds of cancer and affect cell proliferation, angiogenesis, pluripotency, invasion, and metastasis. Although studies have confirmed this connection between IncRNAs and OSCC development, the exact mechanisms of how IncRNAs contribute to OSCC remain unclear (5).

Recent studies have identified ZFASI as a new cancerrelated lncRNA $(12,13)$. Its expression extensively increases in different human cancer types and is related to several 
clinicopathological characteristics and prognostic outcomes. Preliminary exploration has been made to determine the exact mechanisms by which ZFAS1 exerts its effects at the molecular level. As a result, ZFAS1 was found to play a role as ceRNA in regulating the expression of specific genes through competing for target miRNAs $(7,8)$. Based on this research, we found a new lncRNA-miRNA-mRNA network, which was closely associated with OSCC development and contributes new knowledge in the elucidation of the molecular mechanism of OSCC progression.

However, numerous issues must be solved. Firstly, given the critical roles of IncRNAs in transcriptional, posttranslational, and epigenetic regulation (14), the entire ZFASI mechanism in OSCC should be explored. As well as being a ceRNA, ZFAS1 can also modulate genes at the transcriptional level (15). In addition, ZFASI was found to participate in certain signal transduction pathways associated with cancer genesis and development, such as the epithelialmesenchymal transition, p53, and Notch signal transduction pathways (16). Secondly, more studies are needed to explore the utility of ZFAS1 in diagnosing and predicting the prognosis of OSCC. With the development of liquid biopsy technology in cancer, some lncRNAs have been found in cancer tissues and body fluids, such as urine and plasma (17). This is also a noteworthy direction, and we may explore the possibility of ZFAS1 being a liquid biopsy marker in OSCC in our subsequent study.

The $m i R-6499-3 p$ expression level and function in OSCC have not been explored yet. But considering studies showing the prognostic value of changed miRNA levels in the blood and saliva of patients with $\operatorname{OSCC}(18,19)$, the value of $m i R$ $6499-3 p$ in OSCC as a noninvasive diagnostic marker deserves further exploration.

CCL5 has a fundamental role in inflammation for recruiting leukocytes into inflammatory sites. But recently, it has also been found that CCL5 can be secreted by cancer cells and appears to have an essential role in cancer. It plays a role as a growth factor in promoting new blood formation and metastasis or stimulating the formation immunosuppressive microenvironment, even being clinically associated with poor prognosis and metastasis formation (20). Furthermore, previous studies have shown that the level of CCR5 (a specific receptor of CCL5) was positively correlated with late clinical stage, lymph node metastasis, low degree of tumor differentiation, short disease-free survival, and relapse of OSCC, and can serve as a marker of poor prognosis $(21,22)$. Therefore, the CCL5CCR5 axis deserves more attention as expression patterns might be combined as prognostic indicators.

\section{Conclusion}

LncRNA ZFAS1 was significantly up_regulated in human OSCC tissues and cells. Silencing ZFASI suppressed cell proliferation, invasion, and migration in vivo and in vitro. ZFAS1 also acts as a molecular sponge for miRNA-6499-3p in OSCC development by modulating the miRNA-6499-3p/CCL5 axis. Collectively, these findings suggest that lncRNA ZFAS1 might be a valuable therapeutic target for patients with OSCC.

\section{Conflicts of Interest}

The Authors declare no competing financial interest.

\section{Authors' Contributions}

HC designed experiments; XLQ and CXL carried out experiments; CXL analyzed the data. XLQ wrote the article. All Authors approved the final article as submitted and agree to be accountable for all aspects of the work.

\section{References}

1 Bray F, Ferlay J, Soerjomataram I, Siegel RL, Torre LA and Jemal A: Global cancer statistics 2018: GLOBOCAN estimates of incidence and mortality worldwide for 36 cancers in 185 countries. CA Cancer J Clin 68(6): 394-424, 2018. PMID: 30207593. DOI: $10.3322 /$ caac. 21492

2 Chi AC, Day TA and Neville BW: Oral cavity and oropharyngeal squamous cell carcinoma - an update. CA Cancer J Clin 65(5): 401-421, 2015. PMID: 26215712. DOI: 10.3322/caac.21293

3 Bloebaum M, Poort L, Böckmann R and Kessler P: Survival after curative surgical treatment for primary oral squamous cell carcinoma. J Craniomaxillofac Surg 42(8): 1572-1576, 2014. PMID: 24636353. DOI: 10.1016/j.jcms.2014.01.046

4 Peng WX, Koirala P and Mo YY: LncRNA-mediated regulation of cell signaling in cancer. Oncogene 36(41): 5661-5667, 2017. PMID: 28604750. DOI: 10.1038/onc.2017.184

5 Zhang L, Meng X, Zhu XW, Yang DC, Chen R, Jiang Y and Xu $\mathrm{T}$ : Long non-coding RNAs in Oral squamous cell carcinoma: biologic function, mechanisms and clinical implications. Mol Cancer 18(1): 102, 2019. PMID: 31133028. DOI: 10.1186/s12943019-1021-3

6 Di Leva G, Garofalo $\mathrm{M}$ and Croce CM: MicroRNAs in cancer. Annu Rev Pathol 9: 287-314, 2014. PMID: 24079833. DOI: 10.1146/annurev-pathol-012513-104715

7 Li X, Luo Y, Liu L, Cui S, Chen W, Zeng A, Shi Y and Luo L: The long noncoding RNA ZFAS1 promotes the progression of glioma by regulating the miR-150-5p/PLP2 axis. J Cell Physiol 235(3): 2937-2946, 2020. PMID: 31535380. DOI: 10.1002/jcp.29199

8 Liu G, Wang L, Han H, Li Y, Lu S, Li T and Cheng C: LncRNA ZFAS1 promotes growth and metastasis by regulating BMI1 and ZEB2 in osteosarcoma. Am J Cancer Res 7(7): 1450-1462, 2017. PMID: 28744396.

9 Aldinucci D, Borghese C and Casagrande N: The CCL5/CCR5 axis in cancer progression. Cancers (Basel) 12(7): 1765, 2020. PMID: 32630699. DOI: 10.3390/cancers 12071765

10 Aldinucci D and Colombatti A: The inflammatory chemokine CCL5 and cancer progression. Mediators Inflamm 2014: 292376, 2014. PMID: 24523569. DOI: 10.1155/2014/292376

11 Walens A, DiMarco AV, Lupo R, Kroger BR, Damrauer JS and Alvarez JV: CCL5 promotes breast cancer recurrence through 
macrophage recruitment in residual tumors. Elife 8: e43653, 2019. PMID: 30990165. DOI: 10.7554/eLife.43653

12 Dong D, Mu Z, Zhao C and Sun M: ZFAS1: a novel tumorrelated long non-coding RNA. Cancer Cell Int 18: 125, 2018. PMID: 30186041. DOI: 10.1186/s12935-018-0623-y

$13 \mathrm{He}$ A, He S, Li X and Zhou L: ZFAS1: A novel vital oncogenic lncRNA in multiple human cancers. Cell Prolif 52(1): e12513, 2019. PMID: 30288832. DOI: $10.1111 /$ cpr.12513

14 Marchese FP, Raimondi I and Huarte M: The multidimensional mechanisms of long noncoding RNA function. Genome Biol 18(1): 206, 2017. PMID: 29084573. DOI: 10.1186/s13059-017$1348-2$

15 Hansji H, Leung EY, Baguley BC, Finlay GJ, Cameron-Smith D, Figueiredo VC and Askarian-Amiri ME: ZFAS1: a long noncoding RNA associated with ribosomes in breast cancer cells. Biol Direct 11(1): 62, 2016. PMID: 27871336. DOI: 10.1186/s13062-016-0165-y

16 Gao K, Ji Z, She K, Yang Q and Shao L: Long non-coding RNA ZFAS1 is an unfavourable prognostic factor and promotes glioma cell progression by activation of the Notch signaling pathway. Biomed Pharmacother 87: 555-560, 2017. PMID: 28081466. DOI: 10.1016/j.biopha.2017.01.014

17 Jiang N, Pan J, Fang S, Zhou C, Han Y, Chen J, Meng X, Jin X and Gong Z: Liquid biopsy: Circulating exosomal long noncoding RNAs in cancer. Clin Chim Acta 495: 331-337, 2019. PMID: 31054913. DOI: 10.1016/j.cca.2019.04.082

18 Momen-Heravi F, Trachtenberg AJ, Kuo WP and Cheng YS: Genomewide study of salivary microRNAs for detection of oral cancer. J Dent Res 93(7 Suppl): 86S-93S, 2014. PMID: 24718111. DOI: $10.1177 / 0022034514531018$
19 Ries J, Baran C, Wehrhan F, Weber M, Neukam FW, KrautheimZenk A and Nkenke E: Prognostic significance of altered miRNA expression in whole blood of OSCC patients. Oncol Rep 37(6): 3467-3474, 2017. PMID: 28498442. DOI: 10.3892/or.2017.5639

20 Allavena P, Germano G, Marchesi F and Mantovani A: Chemokines in cancer related inflammation. Exp Cell Res 317(5): 664-673, 2011. PMID: 21134366. DOI: 10.1016/j.yexcr.2010.11.013

21 Domingueti CB, Janini JB, Paranaíba LM, Lozano-Burgos C, Olivero P and González-Arriagada WA: Prognostic value of immunoexpression of CCR4, CCR5, CCR7 and CXCR4 in squamous cell carcinoma of tongue and floor of the mouth. Med Oral Patol Oral Cir Bucal 24(3): e354-e363, 2019. PMID: 31011147. DOI: $10.4317 /$ medoral.22904

22 González-Arriagada WA, Lozano-Burgos C, Zúñiga-Moreta R, González-Díaz P and Coletta RD: Clinicopathological significance of chemokine receptor (CCR1, CCR3, CCR4, CCR5, CCR7 and CXCR4) expression in head and neck squamous cell carcinomas. J Oral Pathol Med 47(8): 755-763, 2018. PMID: 29797610. DOI: 10.1111/jop.12736

Received May 15, 2021

Revised July 25, 2021

Accepted July 27, 2021 